\title{
Risk Assessment Stratification Protocol (RASP) to help patients decide on the use of postexposure prophylaxis for HIV exposure
}

\author{
Les Vertesi, MD, MHSc
}

See also pages 35, 36 and 38.

\section{Introduction}

All risks are relative. The response of most people to risks however, comes not from rational processes, but from fear. Situations in which HIV prophylaxis must be considered put emergency physicians into a difficult position. Guidelines are fine in theory, but in practice, people exposed to something as fear-inspiring as HIV are usually not in a position to make logical choices. The Risk Assessment Stratification Protocol (RASP) (Fig. 1) uses the principles of Bayesian analysis to give people a way to make decisions under these circumstances, by putting their risk into perspective alongside risks that we all take in our everyday lives. Table 1 is a useful guide to help patients understand what various levels of risk really mean.

There is one important caveat. Probabilities look like numbers and therefore tend to be used as measurements. They are not, however, really numbers, but estimates, which means they cannot have exact values. To illustrate, probabilities of $1 / 1000$ and 1/1100 are for all intents and purposes the same thing. When discussing probabilities of this nature, only large differences are important. This protocol assumes that the minimum relevant difference for decision-making purposes is one order of magnitude (a factor of 10). So even if some of the values in the RASP formula are not precise, it makes little difference because they would need to be out by a factor of 10 to substantially alter any decisions.

\section{Using the RASP}

Steps A, B, and C assess the probability of exposure to the virus by assigning a score to each of the major risk fac- tors. Bayes' theorem tells us that probabilities that occur in sequence are multiplied together to give a net probability, so the product of these 3 scores $(\mathrm{A} \times \mathrm{B} \times \mathrm{C})$ forms the denominator for the "Basic Risk." Step D gives us a multiplier, or numerator for the Basic Risk. Together they give us the "Total Risk" of contracting HIV from the given exposure. The values used in Steps A to D have been adjusted to reflect as closely as possible the actual experience in an average Canadian community. In places with a different prevalence of disease, these would need to be modified.

\section{Example 1}

A hospital worker is pricked by a needle from a known HIV carrier who does not have clinical AIDS. In this case, value $\mathrm{A}=10$, value $\mathrm{B}=1$, and value $\mathrm{C}=100 . \mathrm{A} \times \mathrm{B} \times \mathrm{C}=$ 1000 so the Basic Risk is $1 / 1000$. Assuming we are dealing with a small-bore $25-\mathrm{g}$ needle, the multiplier is 3 , so the Total Risk is $3 / 1000$ or approximately 1 in 300 . This is a small risk, but definitely worth treating.

\section{Example 2}

A hospital worker is pricked by an old needle from a hospital garbage tray of unknown age, but probably at least 24 hours old. The wound is small, and there is no bleeding. In this case value $A=1000$, value $B=100$ and value $C=$ 200. The Basic Risk then is 1 in 20000,000 . Even if this is a large-bore 18-g needle (modifier value $=5$ ), the Total Risk is still only 1 in 4 million, about equal to your lifetime risk of being on a bridge when it collapses. This exposure is not worth treating.

The treatment thresholds suggested in Table 2 are merely suggestions but they follow the principle that if

From the Royal Columbian Hospital, New Westminster, BC

Submitted: Nov. 12, 2001; final submission: Feb. 11, 2002; accepted: Mar. 1, 2002 
Step A. Identify source population (choose one):

$\underline{\text { Score }}$

Known HIV carrier:

Acute AIDS illness*

Asymptomatic

Unknown HIV status:

High-risk situation

Low-risk situation (other)

A value $=$

Step B. Identify inoculum type (choose one):

Fresh blood

Body fluids at risk (e.g., semen)

Dried old blood

Low-risk secretions (tears, saliva, urine)

B value $=$

\section{Step C. Identify method of transmission (choose one):}

Intravenous

Deep intramuscular

Deep transcutaneous with visible bleeding at site

Superficial transcutaneous with no visible bleeding

Total score $(Z)=A \times B \times C=$ AND Basic risk $=1 / Z=$

\section{Step D. Estimate volume of inoculum (choose one):}

Modifier

Massive (e.g., transfusion)

Measurable ( $>1 \mathrm{~mL}$ )

Moderate (large-bore hollow needle $>22 \mathrm{~g}$ )

Small (small-bore hollow needle $<22 \mathrm{~g}$ )

Trace surface only (e.g., suture needle)

\section{Total risk $=$ Basic risk $\times$ Modifier $(D)=$}


Table 1. Risks in everyday life

Risk of dying in the next 12 months

Overall risk of dying in the next 12 months (all causes) $\quad 1 / 3000$

Specific causes of death in the next 12 months

- from a lightning strike $\quad 1 / 2000000$

- in an accident in your bathtub or shower $\quad 1 / 1000000$

- from a previously unknown allergy to a prescribed drug $\quad 1 / 1000000$

- by choking to death on food

- in a bicycle accident (if you own a bicycle)

- from toxic shock if you use tampons

- by drowning

- from a fire

- $\quad$ as a pedestrian hit by a car or truck

$1 / 160000$

$1 / 130000$

$1 / 100000$

$1 / 50000$

$1 / 50000$

$1 / 40000$

- $\quad$ in a work-related accident (office workers)

$1 / 37000$

- from a fall

$1 / 20000$

- $\quad$ in a work-related accident (overall)

$1 / 11000$

- by being murdered

$1 / 11000$

- $\quad$ while jogging (average $2 \mathrm{~h} / \mathrm{wk}$ )

$1 / 10000$

- in a road accident

$1 / 6000$

- from any kind of accident

$1 / 3000$

\section{Other risks}

- $\quad$ risk of dying on your next commerical jet flight

$1 / 5000000$

- lifetime risk of being on a bridge when it collapses

$1 / 4000000$

- risk of dying if you get influenza

$1 / 5000$

- $\quad$ risk of being diagnosed with cancer in the next 12 months (overall death rate $50 \%$ )

$1 / 3600$

- $\quad$ risk of being diagnosed with lung cancer in the next 12 months if you are (or were) a smoker (overall death rate about $90 \%$ )

$1 / 250$

- $\quad$ risk of having a heart attack in the next 12 months if you are over 35 years of age

$1 / 77$

Adapted from Laudan L. The Book of Risks: Fascinating Facts About the Chances We Take Every Day; John Wiley \& Sons Inc.; 1994.

Table 2. Risk level and treatment recommendation

\begin{tabular}{ll} 
Risk level & Suggested treatment \\
\hline$<1 / 1000$ & Definitely indicated \\
$1 / 1000-1 / 10000$ & Recommended but optional \\
$1 / 10001-1 / 100000$ & Optional but not recommended \\
$>1 / 100000$ & Not indicated \\
\hline
\end{tabular}

something reasonable can be done to minimize risks that are greater than those encountered in daily life, it should be done. For example, our chance of being hit and killed by a car may not be enough to fret about, but it is certainly enough for us to take reasonable precautions, such as using crosswalks as long as they are not too far out of our way. On the other hand, trying to take precautions against being struck by lightning makes no sense because that may involve actions that are at least as risky as the problem we are trying to avoid. Antiretroviral therapy is not without side effects, and even if these are not lethal, they are frequent enough to make compliance an issue. By giving the risk of HIV exposure a numeric instead of a Yes/No value, patients gain the ability to make reasoned choices particular to their own situation, should they choose to do so.

Competing interests: None declared.

Correspondence to: Dr. Les Vertesi; les@ vertesi.com 